\title{
Identité sociale médiatisée à l'exemple de la presse française et polonaise
}

\author{
Mediatized social identity. \\ The case of the French and Polish press
}

\author{
Jolanta Dyoniziak \\ Université Adam Mickiewicz \\ joladyo@amu.edu.pl
}

\begin{abstract}
The article follows the trend of contemporary discursive research by approaching the problem of information media coverage. The main purpose is to describe discursive mechanisms responsible for creating conceptual social space necessary to perception process by individuals of a given society (social identity). The author presents a study of numerous discursive phenomena ordering them according to particular pragmatic objectives of media strategies, such as creating community environment, creating representation of social events, legitimacy of created information. The analysis is based on Polish and French informative press.
\end{abstract}

Keywords: press discourse, news media, media coverage information, social identity

\section{OỦ SOMMES-NOUS, OU QUEL EST LE CADRE THÉORIQUE DE NOTRE ÉTUDE?}

Le présent article se situe dans le cadre des études sur la communication, notamment celles qui visent à mettre en lumière la stratégie de la médiatisation de l'information. Le phénomène évoqué étant bien complexe, ses multiples facettes suscitent un vrai débat scientifique qui conduit à l'élaboration des analyses pluridisciplinaires (entre autres : Gérard, 1999 ; Jairo, 2010 ; Marchetti, 2002 ; Peraya, 1999). La nôtre engage la perspective discursive dans le traitement de l'information (Charaudeau, 2000). La problématique mentionnée est liée à l'énonciation qui s'opère dans le cadre général de communication médiatique, régie en détails par le cadre de l'énonciation journalistique (Charaudeau, 2006). 
La socialisation des individus se fait entre autres par le biais des médias qui lancent des représentations des faits ainsi que des rôles sociaux ${ }^{1}$. L'étude des données recueillies durant la recherche sur la presse nous a fait voir qu'au cours du processus de la médiatisation de l'information, une stratégie discursive est incontestablement mise en œuvre afin de construire une image sociale dont le rôle est fortement identitaire. Il y est question d'une pratique discursive complexe, visant à créer, par le biais d'une série continue de mécanismes divers, un univers sémantique commun aux locuteurs, celui des valeurs et des raisonnements allant à la rencontre de l'espace social. Cela permet à l'individu de participer à la vie communautaire en tant que membre et de s'identifier en fonction des définitions sociales admises. Selon Augé, l'univers sémantique ainsi créé est constitutif de chaque définition individuelle (1992).

La stratégie discursive dont il est question est, entre autres, mise en œuvre grâce à la prédication suivant les apparences logiques du raisonnement approuvant l'esprit ainsi que l'intuition commune. Molinié (1992) parle à ce propos de la formation consciente du champs social dans le discours. Charaudeau (2003) insiste sur la mise en scène de la fiction issue de la prise en charge des événements sociaux. Tsoukala parle d'une structuration de la réalité au lieu de la refléter, d'une représentation sociale (Tsoukala, 2002). Elle est incontestablement au fondement du processus identitaire puisqu'elle permet la construction sociale. Autrement dit, l'identité sociale issue du processus de la médiatisation de la réalité constitue un univers sémantique commun à un groupe d'individus plus au moins restreint suivant le paramètre de dimension décrit plus haut, et s'inscrit dans les mécanismes structurant la cognition sociale. Les représentations sociales (RS) issues du processus de médiatisation, ayant leurs références et leurs répères dans l'espace social, constituent des invariants, des normes partagées au sein d'une société, des jugements de valeur. Selon Charaudeau, l'identité sociale a cette particularité d'être nécessairement reconnue par les autres et elle est posée sur un jugement de valeur. «La façon de raconter s'appuie davantage sur des valeurs d'affect socialement partagées car il s'agit de faire ressentir certaines émotions " (Charaudeau, 2009: 22). Le processus évoqué a un caractère continu, la définition de l'univers événementiel est effectuée de façon permanente. Les médias, quand ils nous informent de la

\footnotetext{
${ }^{1}$ La définition sociale s'effectue par rapport à une unité physique de l'étendue, celle-ci peut être minimale (approche micro) dans le cas des identités propres à certains milieux sociaux, p.ex. régionaux ou professionnels. Elle peut aussi concerner le fonctionnement d'une société à l'échelle nationale, de même que prendre une dimension macro au moment où le sentiment de l'identité sociale régit la vie des nations au niveau international. Cette dernière dimension apparaît actuellement avec une fréquence élevée en raison des mécanismes mettant en marche le systèmemonde, appelé aussi mondialisation.
} 
réalité, ils nous en donne une définition (voir le paragraphe 3.2). Celle-ci admet une interprétation bien que les médias visent à l'objectivité. Ainsi, l'identité sociale à laquelle la médiatisation contribue finalement résulte d'une interactivité incessante entre les savoirs personnels et ceux qui ont leur origine socialement reconnue.

La mise en marche de la stratégie évoquée est directement liée à l'autorité dont disposent les médias, y compris la presse, au sein d'une nation. Ils ont le privilège de produire des représentations sociales (RS) et disposent d'une variété en genre ainsi qu'en nombre des canaux médiatiques appropriés. Ils ont un rôle énonciatif privilégié, socialement sanctionné sous forme $d$ '" un ethos prédiscursif » (Perrin, 2009) et inscrit dans le contrat de communication médiatique (Charaudeau, 2006).

\section{IDENTITÉ SOCIALE MÉDIATISÉE (LE CAS DE LA PRESSE) : QUESTION DU MODÈLE DISCURSIF OU EN QUOI CONSISTE LA MÉTHODOLOGIE ADOPTÉE?}

L'analyse de la médiatisation de l'identité sociale à l'exemple de la presse nous a permis de poser une thèse selon laquelle le processus mentionné réside en un ensemble de mécanismes discursifs coordonnés qui prennent manifestement la forme d'une stratégie. La réalisation de celle-ci se fait par étapes et elle consiste à atteindre trois objectifs principaux qui sont les suivants:

1) création du monde communautaire où l'enjeu de "crédibilité démocratique » (Charaudeau 2003, 2006) reste supérieur par rapport à d'autres visées pragmatiques,

2) interprétation du monde expérimenté afin de lancer une représentation sociale,

3) légitimation de la définition sociale faite où l'enjeu de légitimité est dominant (Charaudeau, 2006).

Pour que le modèle présenté ci-dessus se matérialise, nous avons adopté une démarche méthodologique en deux étapes successives. D'abord, nous avons fait une recherche théorique visant à élaborer le modèle préliminaire de la stratégie discursive mise en œuvre afin de médiatiser l'identité sociale. Plusieurs travaux théoriques analysés nous ont inspiré dans notre démarche, ceux qui sont de nature générale (Charaudeau, 2000 ; Maingueneau, 2002 ; Moirand, 2007), aussi ceux qui traitent des questions détaillées concernant la problématique évoquée (entre autres : Adam, 1997; Adam, Lugrin, 2006 ; Charaudeau, 2003, 2006 ; Koren, 2006 ; Moirand, 2006). Ainsi sommes-nous arrivée à élaborer un modèle discursif que nous avons ensuite appliqué à l'étude du discours journalistique 
réalisé par la presse électronique française et polonaise (ce modèle est présenté ci-dessus, voir les points : 1, 2, 3). On a créé un corpus illustrant la richesse des procédés discursifs mis en œuvre afin de réaliser les objectifs explicités. Les titres mis à l'examen font partie de la presse quotidienne et ils sont les suivants : Libération, le Monde, le Figaro, Gazeta Wyborcza, Rzeczpospolita, Nasz Dziennik. La période analysée englobe les deux dernières décennies (les années : 2000-2014). Nous sommes consciente que le modèle discursif proposé dans le présent travail n'est certainement pas complet et que le problème posé exige une continuation des recherches. Pourtant, il permet d'aborder préliminairement le sujet et d'inciter les recherches dans la problématique évoquée.

\section{IDENTITÉ SOCIALE MÉDIATISÉE : STRATÉGIE DISCURSIVE MISE À L’EXAMEN}

\subsection{CRÉATION DU MONDE COMMUNAUTAIRE}

Le principe de la première étape consiste en une mise en œuvre d'un ensemble de pratiques discursives afin de créer le sentiment d'appartenance communautaire chez les locuteurs. La création du monde communautaire est indispensable du point de vue du locuteur ainsi que de celui du récepteur puisqu'elle contribue à la légitimité ainsi qu'à la crédibilité des informations divulguées (Charaudeau, 2006). Cela se réalise, entre autres, par :

- la prise de contact avec le locutaire de façon explicite,

- la prise de contact avec le locutaire de façon implicite,

- la création des apparences du contact communicationnel direct.

\subsubsection{PRISE DE CONTACT AVEC LE LOCUTAIRE DE FAÇON EXPLICITE}

Parmi les techniques les plus fréquemment utilisées, il faut souligner l'emploi de l'adjectif possessif notre ainsi que nos (voir ex. 1-3), le recours à d'autres adjectifs du type : commun (voir ex. 4-5), la formulation des questions (voir ex. 6-9), surtout sous la forme d'un titre, le recours aux injonctions à la $1^{\text {ère }}$ personne du pluriel. Toutes les techniques explicitées contribuent à l'intégration de l'allocutaire dans une communauté qui selon Koren est "[...] présentée d'emblée comme consensuelle » (Koren, 2006 : 101). Parmi eux, il faut souligner les constructions interrogatives, surtout leur variante rhétorique (voir ex. 9). Cette dernière a un fort potentiel pragmatique, elle oblige le lecteur à accepter la réponse puisque celle-ci est déjà socialement sanctionnée (Charaudeau, 2006). 


\section{Exemples :}

1. Nasz Niemiec Albrecht Lempp ${ }^{2}$ /gazetawyborcza.pl, 26.11.2012

2. Défendre les droits de l'homme à Kiev et à Pékin, c'est notre intérêt /Le Monde.fr, 11.03. 2014

3. L'économie collaborative s'invente en France: gardons notre avance /Le Monde.fr, 6.03. 2014

4. Wspólna sprawa: Kijów - Warszawa! ${ }^{3}$ /Gazeta.pl, 30.01.2014

5. La politique agricole commune est l'affaire de tous /Le Monde, 8.05.2009

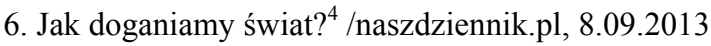

7. Czy wolno nam milczeć? ${ }^{5} /$ naszdziennik.pl, 20.08.2013

8. Les Roms doivent-ils vivre dans l'indignité ? /Libération, 17.10.2013

9. Czy Polak w ogóle potrafi być szczęśliwy? /Gazeta Wyborcza, 21.02.2014

\subsubsection{PRISE DU CONTACT AVEC LE LOCUTAIRE DE FAÇON IMPLICITE PAR DES RÉFÉRENCES À L'ESPACE SÉMANTIQUE COMMUN}

Le journaliste énonciateur met en marche des opérations de référence indirecte (Maingueneau, 2002). Le phénomène se lie au problème de l'interdiscursivité et de l'intertextualité (Hébert, Guillemette, 2009). Le processus de nomination admet une inférence qui s'opère dans la mémoire discursive, celle-ci régit le sujet énonciateur, l'énoncé ainsi que d'autres facteurs contribuant à une activité discursive (Moirand, 2006b, 2007 ; Possenti, 2011). Quant à l'analyse du processus de nomination, il y entre en jeu les unités dénominatives issues de l'activité néologique (ex. 14), surtout celles qui ont été détournées ou contaminées (ex. 12 et 13), les emplois antonymiques, comme par exemple l'oxymore (ex. 15, 16, 17), les emplois métaphoriques des noms propres, les comparaisons véhiculant un contenu sémantique implicite comme par exemple l'amalgame (ex. 10 et 11). Tous les procédés dénominatifs énumérés atteignent particulièrement les titres, le nombre des pratiques linguistiques de nomination explicitées y étant bien élevé par rapport au texte qui suit. L'effet pragmatique consistant à situer le locutaire dans l'espace commun présenté comme consensuel (Koren, 2006), par le fait d'engager celui-ci à faire des inférences sur cet espace, devient

\footnotetext{
${ }^{2}$ Albrecht Lempp, notre Allemand (notre traduction).

${ }^{3}$ fr. Affaire commune : Kiev - Varsovie (notre traduction).

${ }^{4}$ fr. Comment rattrapons-nous le monde ? (notre traduction).

${ }^{5}$ fr. Pouvons-nous rester silencieux ? (notre traduction).

L'article parle des problèmes financiers des personnes qui s'occupent des malades. On leur a enlevé des prestations sociales. La situation a eu lieu en Pologne.

${ }^{6}$ fr. Le Polonais peut-il être heureux ? (notre traduction).
} 
incontestablement plus grand quand il relève de la structure textuelle d'ouverture. Le titre a une place énonciative privilégiée dans ce qu'Adam appelle une « hyperstructure » journalistique (Adam, Lugrin, 2006).

Au niveau de la structure textuelle, c'est entre autres l'anaphore associative qui peut amener au même effet pragmatique. Elle ne reprend pas du déjà dit, mais repose sur des implications lexicales, celles-ci ne relèvent pas du cotexte, elles sont partagées par un ensemble d'individus formant une communauté (Kleiber, 2001).

A titre d'illustration, nous allons décrire quelques procédés explicités cidessus. Commençons par le mécanisme logico-rhétorique d'amalgame (voir ex. 10 et 11). Il repose sur une analogie référentielle que les linguistes qualifient de « fallacieuse » (Matar, Chauvin-Vileno, 2006), ou « [...] pernicieuse et malhonnête au regard de l'éthique de l'information» (Charaudeau, 2006). Il contribue à l'actualisation d' " une mémoire globale, non-discriminante» par le recours fréquent à « une émotion interprétative », et élimine « un effort d'analyse » au cours du processus inférentiel (Charaudeau, 2006). Nos exemples impliquent à chaque fois une équivalence entre deux référents : Turquie et Chine (ex. 10), Poutine et tsar (ex. 11). On ne peut pourtant négliger la force discursive de l'amalgame ainsi que son fondement cognitif, celui-ci constituant un mélange de référents nécessairement partagés au sein d'une communauté (Doury, 2003). Les comparaisons effectuées contribuent au processus identitaire sur le plan social, la mise de l'énonciateur et du locutaire dans le même espace culturel amène au rapprochement de ceux-ci et de même, réduit la distance imposée par le dispositif mis en marche ainsi que par le genre du discours exercé.

Le même effet pragmatique est provoqué par les procédés contribuant à l'intertextualité (entre autres: les détournements, les défigements, certaines formes néologiques, voir les ex. 12,14). Les mots qui sont actuellement mis dans les pages reprennent ceux qui ont été déjà dit, ils réfèrent aux énoncés déjà faits et se situent dans un espace de relations intertextuelles et interdiscursives complexe (Moirand, 2006b ; Possenti, 2011). Ainsi pouvons-nous admettre qu'il s'agit des formes de «particitation» fonctionnant «[...] comme un régime spécifique de citation $[\ldots] »$. Ce sont certainement «[...] des énoncés autonomisés qui font partie du Thésaurus d'un groupe socio-linguistique donné dont la simple évocation garantit le sentiment d'appartenance » (Adam, Lugrin, 2006 : 132). Les exemples : 12 et 14 illustrent le phénomène évoqué. L'énoncé 12 est construit d'un mot-valise (heretyk + heteroseksualny, fr. un hérétique + un hétérosexuel, les deux lexèmes forment un néologisme: un hétérique) qui remplace le mot heretyk (fr. un hérétique) dans la collocation inwazja heretyków (fr. invasion des hérétiques). L'opération lexicale effectuée est réussie au moment où le récepteur sait la décoder de façon correcte ainsi que déduire son 
potentiel pragmatique. Celui-ci nous amène dans le cas du titre évoqué au jugement de valeur négatif. L'exemple 14 constitue une expression contaminée (E1) qui réfère à la version de départ (E0) : wyszło szydło z worka employée pour dire que le secret est dévoilé. Le titre réfère à deux concepts : le premier biblique du paradis perdu et le second du paradis fiscal.

Efficaces sont aussi les figures d'opposition. Les exemples 15, 16 et 17 illustrent la mise en œuvre de la figure de l'oxymore, celui-ci introduisant une relation asémantique au niveau des concepts actualisés en discours. Ainsi se produit-il un effet d'incongruité conceptuelle qui provoque un sentiment de déviance cognitive chez le locutaire. L'oxymore joue sur l'espace sémantique commun et répétitif au niveau de l'usage social. Il met en œuvre des processus cognitifs liés à la violation des contraintes conceptuelles (Reboul, 1991; Wołowska, 2011). Un sème présent dans le sémème-type (ST) est suspendu de la configuration du sémème-occurrence ( $\mathrm{SO}$ ) en raison d'une interprétation contextuellement correcte. Ainsi les SO explicités (voir ex. 15-17) subissent une reconfiguration sémique dans les contextes évoqués: la fascisation est dépourvue du trait /non-humanitaire/ (voir ex. 15), l'espion subit l'action conventionnellement attribuée au patient (voir ex. 16), la république française ne jouit plus du principe d'égalité, ni de tolérance (voir ex. 17)

10. Turquie, une « Chine » à nos portes ${ }^{7} /$ le Journal du Dimanche, 10.10.05 (Moirand, 2006a)

11. Les Russes aiment leurs tsars et Poutine en est un /Le Monde, 8.12.2011

12. Inwazja heteryków /Newsweek, 28.07.2002

13. Wyszło szydło z raju ${ }^{8} /$ gazetawyborcza.pl, 5.04.2013

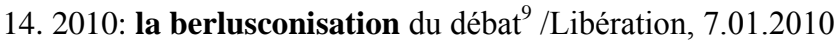

15. Faszyzacja humanitarna ${ }^{10}$ /naszdziennik.pl, 19.07.2013

16. NSA, L'espion espionné /Libération, 14.02.2014

17. République acide pour les Roms /Libération, 11.02.2014

${ }^{7}$ L'article parle de la Turquie en tant que nouvelle puissance économique qui affole les investisseurs étrangers. L'exemple est cité par Moirand (2006a).

${ }^{8}$ Le titre implique l'expression wyszło szydto z worka, en fr. ce n'est plus un secret maintenant, laisser sortir le chat. L'article parle du déversement de plus de deux millions de documents provenant des paradis fiscaux, ce qui a dévoilé les intérêts secrets des Crésus de ce monde.

${ }^{9}$ L'article commente le changement de ton politique fait par Sarkozy. Selon l'auteur, à quelques semaines des élections régionales, ultimes élections directes avant la phase présidentielle, le chef de l'Etat se pose en rassembleur, il reprend une pose plus consensuelle et plus ouverte.

${ }^{10}$ fr. Fascisation humanitaire (notre traduction). 


\subsubsection{CRÉATION DES APPARENCES DU CONTACT COMMUNICATIONNEL DIRECT}

Cela concerne uniquement la presse électronique car il s'agit de la possibilité donnée au récepteur de faire un commentaire au-dessous de l'article. Quant à la presse écrite, l'intervention indirecte du locutaire n'est pas possible, aussi donne-t-on des apparences du contact par la mise des pages nommées: Opinions, Débats ou Courrier, jugées par certains « démocratiques » (MartinCastelnau, 2005) et destinées aux commentaires élaborés par les auteurs choisis. Dans les deux cas, il s'agit de la structure textuelle appelée « épitexte » circulant autour du texte et comprenant les commentaires qui ne font pas partie du même ensemble matériel (Adam, 1997).

Il est aussi important de noter la réduction de la distance énonciateur récepteur par le recours à l'oralité. Perrin (2009) parle même de l'ethos du parler direct dont la force pragmatique contribue à la création d'une fiction énonciative régissant la presse écrite. Le style oral instaure au sein de l'écrit journalistique une forme de scénographie de l'interaction face à face avec le locutaire.

\subsection{MISE EN SCÈNE DES REPRÉSENTATIONS SOCIALES DE LA RÉALITÉ}

Les médias ont un rôle central dans la définition de l'espace social (Charaudeau, 2000). Ils fonctionnent d'après le paradigme constructiviste qui admet la dynamique cognitive dans la construction de la réalité médiatique. «La communication médiatique est $[\ldots]$ considérée comme système de production symbolique, où se produit un sens par la création et la manipulation de signes» (Chaniac, 1998: 95). Chaque évolution de l'image socialement constituée tient pour une bonne part aux discours que les médias émettent (Maingueneau, 2002). La dénomination qui s'y effectue en tant que mode de donation du référent contribue à une interprétation de l'espace social, ce qui a pour but d'éveiller le débat démocratique au sein des communautés. Cela est beaucoup plus pertinent lorsque l'interprétation proposée suscite des émotions. L'interprétation explicitée peut s'effectuer par le fait de véhiculer des points de vue. Selon Rabatel et Chauvin-Vileno «Le point de vue correspond à des manières de voir, qui peuvent s'exprimer à travers le choix de tel mot, ou irriguer de vastes portions discursives. Ces points de vue reposent sur des cadres notionnels plus ou moins élaborés, qui jouent le rôle de site cognitif et de cadre axiologique » (2006: 21). Grize (2005) parle de «l'éclairage» donné par les opérations de référence auxquelles le texte contribue. L'éclairage « [...] cherche à conduire les lecteurs à se construire une opinion, guidés en cela bien évidemment par l'univers que lui a proposé l'énonciateur » (Moirand, 2006b : 53). Le procédé constitue indubitablement un élément de la démarche permettant 
au journaliste-énonciateur de faire un positionnement discursif (Charaudeau, 2006).

Parmi les procédés dénominatifs qui sont particulièrement aptes à l'enjeu interprétatif, on distingue :

- Dénomination prédicative de type comparatif : Paul est un Tarzan (voir les ex. 10 et 11)

Selon Maingueneau (2002), l'énonciateur y met en œuvre certaines propriétés attachées communément par l'opinion au référent second (ici Chine et tsar, ex. 10 et 11), le processus interprétatif n'actualise pas le cotexte, l'inférence résulte d'une simple comparaison explicite qui peut contribuer à un jugement de valeur.

- Anaphore nominale infidèle ou un SN en fonction d'apposition

Puisque l'anaphore constitue la reprise d'un référent dans le texte ou sa redénomination (Theissen, 1997), elle peut contribuer à ce qu'on le voit modifié au niveau de son profil conceptuel. Ainsi, les reprises anaphoriques sont particulièrement sensibles à toutes sortes d'interprétations et elles peuvent véhiculer des jugements de valeur, ce que suggèrent les exemples suivants :

18. Angela Merkel, cesarzowa Europy ${ }^{11}$ /gazeta wyborcza, 14.02.2014

19. IVG, le grand retour en arrière espagnol /Libértion, 23.12. 2013

20. Le racisme, l'inégalité avant tout/Libération, 16.02.2014

21. Ianoukovitch, voyou devenu président /Libération, 20.02.2014

- Emploi générique ou plus fréquent, emploi généralisant

On remarque le phénomène de la modulation sur la généralisation particulièrement fréquente dans le discours quotidien. Le procédé a lieu quand "l'énonciateur de loi négocie la forme généralisante de son énoncé pour la rendre plus acceptable à son auditoire » (Ali Bouacha, 1993 : 314). Ainsi, dans le discours journalistique, les énoncés généralisants qui admettent la lecture générique (voir les ex. 22 et 23) sont-ils dominants. Parmi les marques morphosyntaxiques saillantes des énoncés évoqués, il faut souligner le recours aux prédicats de classe ainsi que l'emploi du présent. Les premiers ne peuvent s'appliquer à un individu, ni à un événement individuel en situant la catégorisation au niveau de base puisque le défini générique singulier et pluriel vise une classe référentielle et non ses occurrences. Le temps présent est le plus apte parmi les temps français à perdre sa référence temporelle, ainsi admet-on une interprétation indépendante des circonstances de l'énonciation (Galmiche, 1985 : 6-7).

\footnotetext{
${ }^{11}$ fr. Angela Merkel, l’impératrice de l’Europe (notre traduction).
} 
22. Les immigrés ne sont pas des criminels /Libération, 7.10.2013

23. Le racisme, l'inégalité avant tout/Libération, 16.02.2014

- Dénomination néologique p.ex. par le défigement (voir ex. 12: Inwazja heteryków $^{12}$ (Newsweek, 28.07.2002)

- Mots affectifs

Le recours aux mots affectifs est fréquent puisqu'il contribue facilement à introduire un jugement de valeur ainsi qu'à faire ressentir certaines émotions. Moirand (2006b) appelle cette forme d'actualisation du référent comme désignations qualifiantes et la met parmi les actes de nomination pragmatiquement efficace.

24. Ianoukovitch, voyou devenu président /Libération, 20.02.2014

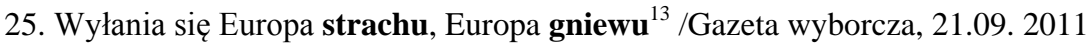

26. Europejska forteca, czyli imigranckie piekło na ziemi ${ }^{14} /$ Newsweek, 14.10.2013

À côté des procédés dénominatifs, on voit d'autres de nature purement discursive comme, par exemple les généralisations discursives (ex. 27). Le phénomène a lieu lorsqu'il y a un décalage énonciatif entre l'étendue de l'information inclue dans l'article et celle suggérée par le titre. Il s'agit de l'attribution à tout un ensemble social d'un trait propre à un individu. Il est lié à la mise en œuvre de la stratégie de dramatisation (Charaudeau, 2006). L'article (ex. 27) informe du procès contre un homme soupçonné d'avoir aspergé en janvier 2014 à Paris le matelas d'un couple de Roms d'un produit corrosif. L'information concerne un événement provoqué par un seul individu, tandis que le titre ouvre la voie à l'interprétation généralisante selon laquelle tous les Français, en plus républicains, sont hostiles aux immigrés Roms.

27. République acide pour les Roms /Libération, 11.02.2014

\subsection{PROCÉDÉS DE LÉGITIMATION DES REPRÉSENTATIONS FAITES}

D'abord, le journaliste-énonciateur jouit d'une autorité qui lui est socialement attribuée en fonction de sa profession, «[...] le sujet qui informe étant légitimé par avance (contrat de communication) [...]» (Charaudeau,

\footnotetext{
${ }^{12}$ L'article parle de Clubs Gay qui vivent l'invasion de jeunes hétérosexuels. L'auteur se pose une question: est-ce une expression de l'acceptation sociale de l'altérité, ou plutôt une manifestation du goût pour l'exotisme?

${ }^{13}$ fr. L'Europe de la peur, de la colère apparait (notre traduction). L'article parle d'un essai politique sur l'évolution de la démocratie en Europe dont Marek Beylin est l'auteur.

${ }^{14} \mathrm{fr}$. Forteresse européenne, enfer des immigrés sur terre (notre traduction).
} 
2006). Cela peut contribuer à la légitimation des propos tenus, surtout au niveau des opérations cognitives effectuées automatiquement par le récepteur. Pourtant sa position énonciative peut devenir encore plus forte lorsqu'il choisit et met en œuvre de façon cohérente des moyens discursifs appropriés à la légitimation. Parmi les plus fréquents, il faut énumérer :

\section{- Recours à la répétition d'une RS}

Il s'agit du phénomène discursif qui peut prendre différentes formes. On peut y noter la citation réciproque, elle apparaît avec une fréquence élevée quand la radio cite la presse, la presse la télévision, et celle-ci parfois la presse (Charaudeau, 2003). La répétition peut avoir sa seconde source dans la présentation cyclique d'un événement, selon Moirand il s'agit d' « un traitement d'un moment discursif particulier qui généralement s'étale sur plusieurs numéros, voire sur plusieurs semaines ou plusieurs mois, et qui revient cycliquement au cours du temps : ainsi, en France, la grippe aviaire a été l'objet de ce genre de pages en octobre 2005, est revenue à la une en janvier - février 2006 lors de la progression du virus vers l'Europe et de la découverte du premier cas en France » (2006a). D'abord, la répétition fréquente des propos supprime la possibilité de recevoir une nouvelle avec un esprit critique, elle automatise la réception qui s'effectue par défaut. Ensuite, elle contribue à la «fidélisation des récepteurs » (Rabatel, Chauvin-Vileno, 2006) à l'information explicitée, on engage ceux-ci au " déroulement » social d'un événement. Selon Charaudeau, «Cette information, répétée de la même façon ou avec des variantes, produit un effet de réification : la nouvelle prend une existence en soi, se trouve par là même authentifiée, se fige et donc s'inscrit de façon indélébile dans la mémoire » (2006).

- Recours à la modalité affirmative

Les auteurs soulignent la modalité d'affirmation en tant que catégorie privilégiée dans la médiatisation de l'information par la presse (Charaudeau, 2006, Moirand, 2006a). «[...] l'énonciateur journalistique cherche à s'effacer derrière un sujet expliquant indéterminé, il n'emploie guère de marques de modalisation du discours, car, aux dires du milieu journalistique elles risqueraient de produire un effet d'incertitude, de doute, contradictoire avec les attentes (une fois de plus supposées) des lecteurs. C'est pourquoi le discours explicatif journalistique se présente sous la modalité de l'affirmation : modaliser serait une preuve de faiblesse au regard de la visée de crédibilité de la machine informative » (Charaudeau, 2006).

- Recours à la polyphonie

Il s'agit de l'hétérogénéité du discours sur le plan énonciatif (Nølke, 2004). Plusieurs voix - autorités sont introduites afin de confirmer les propos dits. La 
stratégie consiste en une donation de valeur de vérité au point de vue présenté et simultanément contribue à l'individualisation du discours, et permet de réaliser l'effacement énonciatif du journaliste. Plusieurs procédés y sont pertinents : le recours aux sondages, aux enquêtes, aux citations, etc., ceux-ci introduits souvent à l'aide du discours indirect. « La mise en scène de ce que l'on appelle le discours rapporté devrait également satisfaire à un principe de distance et de neutralité qui oblige le rapporteur journaliste à s'effacer, et dont la marque essentielle est l'emploi des guillemets encadrant le propos rapporté. C'est là encore se soumettre à l'enjeu de crédibilité [...]» (Charaudeau, 2006). Parmi les procédés contribuant à la citation, fréquentes sont les citations réciproques résultant d'un échange d'information entre plusieurs agents médiatiques (voir le paragraphe 3.3 : recours à la répétition d'une RS). Il faut signaler encore que les sondages et les enquêtes mis dans les pages peuvent avoir une nature autoévaluative quand ils portent sur les rapports médias-opinion publique. Les deux derniers procédés y paraissent bien pertinents puisqu'ils servent à conforter l'opinion publique ainsi qu'à porter son attention sur le bien fondé de l'information médiatique (Charaudeau, 2003).

\section{CONCLUSION}

La stratégie discursive mettant sur la scène publique une identité sociale est bien complexe, elle consiste en la réalisation de plusieurs objectifs comme : création de l'espace commun, interprétation de la réalité sociale aboutissant à la mise en public des représentations sociales, mise en légitimation des représentations faites.

La présente analyse, quoique préliminaire, montre que la médiatisation de l'information vue dans sa totalité en tant qu'ensemble de processus multiples et continus, aboutit à la construction des images sociales. Celles-ci influent certainement la perception des individus - membres d'une communauté. L'espace conceptuel ainsi créé, référant aux événements inhérents à la vie sociale, constitue un savoir partagé décidant de notre perception identitaire. 


\section{BIBLIOGRAPHIE}

ADAM, J.-M. (1997): «Unités rédactionnelles et genres discursifs : cadre général pour une approche de la presse écrite ». Pratiques, 94, 3-18.

ADAM, J.-M., LuGRIN, G. (2006): «Effacement énonciatif et diffraction co-textuelle de la prise en charge des énoncés dans les hyperstructures journalistiques ». Semen, 22, 127-145. URL : http://semen.revues.org/4381, consulté le 05 mars 2014.

Ali BouachA, A. (1993): «L'énoncé généralisant: statut et enjeux ». In : Ch. Plantin, (ed.), Lieux communs, topoï, stéréotypes, clichés. Paris : Kimé, 312-323.

Auge, M. (1992): Non-lieux, introduction à une anthropologie de la surmodernité. Paris : Le Seuil.

CHANIAC, R. (1998): «Le discours d'information médiatique. La construction du miroir social, Patrick Charaudeau ». Langage et société, 85, 95-102.

Charaudeau, P. (2000): Le discours d'information médiatique. La construction du miroir social. Paris : Nathan.

Charaudeau, P. (2003): «Les médias, un manipulateur manipulé ». In : Ph. Ricalens, La manipulation à la française. Paris : Economica. URL : http://www.patrick-charaudeau. com/Les-medias-un-manipulateur.html, consulté le 17 mars 2014.

Charaudeau, P. (2006): «Discours journalistique et positionnements énonciatifs. Frontières et dérives ». Semen, 22. URL : http://semen.revues.org/2793, consulté le 04 mars 2014.

Charaudeau, P. (2009): "Identité sociale et identité discursive. Un jeu de miroir fondateur de l'activité langagière ». In: P. Charaudeau (ed.), Identités sociales et discursives du sujet parlant. Paris : L'Harmattan, 15-29.

Doury, M. (2003): «L'évaluation des arguments dans les discours ordinaires: le cas de l'accusation d'amalgame ». Langage et Société, 105, 9-37.

GaLmiche, M. (1985): «Phrases, syntagmes et articles génériques ». Langages, 79, 2-39.

GERARD, L. (1999): La société de communication, Une approche sociologique et critique. Paris : PUF.

GRIZE, J.-B. (2005): « Le point de vue de la logique naturelle. L'argumentation aujourd'hui ». In : M. Doury, S. Moirand (ed.), Positions théoriques en confrontation. Paris : Presses Sorbonne Nouvelle, 35-43.

HeBert, L., GuillemetTe, L. (2009): Intertextualité, interdiscursivité et intermédialité. Québec : Presses de l'Université Laval.

JAIRO, F. (2010): " La médiatisation : de la production à la circulation des nouvelles ». Les Enjeux de l'information et de la communication, 1, 121-143. URL : www.cairn.info/revue-les-enjeuxde-l-information-et-de-la-communication-2010-1-page-121.htm

KLEIBER, G. (2001): L'anaphore associative. Paris : PUF.

Koren, R. (2006): «La responsabilité des Uns dans le regard des Autres : l'effacement énonciatif au prisme de la prise de position argumentative ». Semen, 22. URL : http://semen.revues. org/2820, consulté le 05 mars 2014.

MaingueneAu, D. (2002): Analyser les textes de communication. Paris : Nathan Université.

MARChetTI, D. (2002): «Sociologie de la production de l'information ». Cahiers de la recherche sur l'éducation et les savoirs, 1, 17-32.

Martin-Castelnau, D. (2005): "Enquête sur les professionnels de la "tribune libre" ». Marianne, 423-424, 90-95.

MataR, S., ChAuvin-Vileno, A. (2006): «Islamalgame, discours représenté et responsabilité énonciative ». Semen, 22, URL : http://semen.revues.org/2804, consulté le 05 mars 2014. 
MoIRAND, S. (2006a): «Responsabilité et énonciation dans la presse quotidienne : questionnements sur les observables et les catégories d'analyse ». Semen, 22. URL : http://semen.revues. org/2798, consulté le 05 mars 2014.

MoIRAND, S. (2006b): «Entre discours et mémoire: le dialogisme à l'épreuve de la presse ordinaire ». Travaux neuchâtelois de linguistique, 44, 39-55.

MoIRAND, S. (2007): Le discours de la presse quotidienne: observer, analyser, comprendre. Paris : PUF.

Molinie, G. (1992): Dictionnaire de rhétorique. Paris : Le Livre de Poche.

Nølke, H., Fløttum, K., Noren, C. (2004): ScaPoLine. La théorie scandinave de la polyphonie linguistique. Paris : Kimé.

Peraya, D. (1999): « Médiation et médiatisation : le campus virtuel ». La Revue, 25, 153-167.

Perrin, L. (2009): "L'ethos de l'oralité comme forme de fiction énonciative dans la presse écrite ». In : M. Burger, J. J. R. Micheli (ed.), Actes du colloque : Le français parlé dans les médias : les médias et le politique. Lausanne. URL : http://www.unil.ch/webdav/site/clsl/ shared/Actes_FPM_2009/PerrinFPM2009.pdf, consulté le 28 mai 2014.

Pineira-Tresmontant, C. (2003): " Patrick Charaudeau, Le discours d'information médiatique. La construction du miroir social ». Mots. Les langages du politique, 72, 181-182. URL: http://mots.revues.org/6763, consulté le 13 mars 2014.

Possenti, S. (2011): "Réflexions sur la mémoire discursive ». Argumentation et Analyse du Discours, 7. URL : http://aad.revues.org/1200, consulté le 17 juin 2014.

Rabatel, A., Chauvin-Vileno, A. (2006): «La question de la responsabilité dans l'écriture de presse ». Semen, 22, 7-29. URL : http://semen.revues.org/2792, consulté le 04 mars 2014.

Reboul, O. (1991): Introduction à la rhétorique. Paris : Presses Universitaires de France.

Theissen, A. (1997): Le choix du nom en discours. Genève - Paris : Librairie Droz.

TsoukALA, A., (2002): "Le traitement médiatique de la criminalité étrangère en Europe ». Déviance et Société, 26, 61-82.

WolowsKa, K. (2011): «La virtualisation contextuelle de traits sémantiques : non-actualisation, délétion ou suspension? ». Texto! Textes \& Cultures, 2. URL : http://www.revue-texto.net/ index.php?id=2848, consulté le 13 avril 2014: 